\title{
Nonlinear Energy Transfer of Drift-Wave Fluctuation in a Cylindrical Laboratory Plasma
}

\author{
Yoshihiko NAGASHIMA, Sanae -I. ITOH ${ }^{1)}$, Shunjiro SHINOHARA ${ }^{2)}$, Masayuki FUKAO ${ }^{3)}$, \\ Akihide FUJISAWA ${ }^{4)}$, Kenichiro TERASAKA ${ }^{2)}$, Yoshinobu KAWAI ${ }^{2)}$, Naohiro KASUYA ${ }^{4)}$, \\ Masatoshi YAGI $^{1)}$, Shigeru INAGAKI ${ }^{1)}$, Takuma YAMADA and Kimitaka ITOH ${ }^{4)}$ \\ Graduate School of Frontier Sciences, The University of Tokyo, Kashiwa 509-5292, Japan \\ ${ }^{1)}$ Research Institute for Applied Mechanics, Kyushu University, Kasuga 816-8580, Japan \\ ${ }^{2)}$ Interdisciplinary Graduate School of Engineering Sciences, Kyushu University, Kasuga 816-8580, Japan \\ ${ }^{3)}$ Myojocho, Uji 611-001, Japan \\ ${ }^{4)}$ National Institute for Fusion Science, Toki 509-5292, Japan
}

(Received 18 July 2008 / Accepted 14 August 2008)

\begin{abstract}
Nonlinear energy transfer of the drift-wave spectrum is investigated experimentally in a cylindrical laboratory plasma. Using the Hasegawa-Mima equation, the nonlinear transfer function of electrostatic potential energy in frequency space is estimated by bispectral analysis. The transfer function derived from the drift-wave spectrum indicates a forward cascading of energy transfer from fundamental to higher harmonic modes.
\end{abstract}

(c) 2008 The Japan Society of Plasma Science and Nuclear Fusion Research

Keywords: drift-wave, Hasegawa-Mima equation, bispectral analysis, nonlinear energy transfer

DOI: $10.1585 /$ pfr.3.056

It is recognized that drift-wave turbulence dominates in magnetized plasmas with a finite density gradient and makes a significant contribution to plasma transport called anomalous transport [1]. Turbulence consists of not only linearly unstable modes but also nonlinearly excited quasimodes. Therefore, to understand turbulence and control transport, research on nonlinear energy transfer of selfregulated turbulence in the phase space (frequency and wave number space) is crucial. Quadratic nonlinearity plays a dominant role on the energy transfer. In this rapid communication, we present a direct estimation of the energy transfer function and direction of energy cascading in the drift-wave spectrum in a laboratory plasma.

Experiments were conducted on a linear device, the Large Mirror Device (LMD) [2,3]. In the LMD, nonlinearity between the drift-wave spectrum and the low-frequency zonal $\boldsymbol{E} \times \boldsymbol{B}$ velocity is investigated intensively [4, 5]. The experimental conditions were; RF power $=2 \mathrm{~kW}$, magnetic field $B=0.12 \mathrm{~T}$, and filling argon gas pressure $\sim 3.5 \mathrm{mTorr}$. The configuration of $B$ converges near the plasma source and is straight in the vessel. Fluctuations are mainly measured with a Reynolds stress probe (RSP) [3]. The RSP has three tungsten electrodes with poloidal or radial distances of $4.4 \mathrm{~mm}$ or $5 \mathrm{~mm}$. The radial wave number $k_{r}$ and the poloidal wave number $k_{\theta}$ are derived from the cross-phase of the floating potential fluctuation $\tilde{\Phi}_{\mathrm{f}}$. The right-handed system is selected in the coordinate.

Figure 1 shows the spectra of $\tilde{\Phi}_{\mathrm{f}}$, auto-power (a), $k_{\theta}$ and $k_{r}(\mathrm{c})$. The spectra are measured at the radial loca-

author'se-mail: nagashima@k.u-tokyo.ac.jp
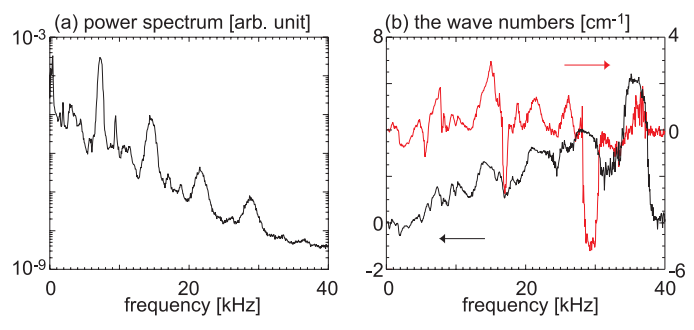

Fig. 1 (a) Auto-power spectrum of the floating potential fluctuation $\tilde{\Phi}_{\mathrm{f}}$. (b) The poloidal wave number $k_{\theta}$ (black), and the radial wave number $k_{r}$ (red) of $\tilde{\Phi}_{\mathrm{f}}$.

tion $r=3.25-3.5 \mathrm{~cm}$ where the inverse of the density scale length and the normalized fluctuation amplitude have maxima. In Fig. 1 (a), a distinct spectral peak at $7-8 \mathrm{kHz}$ (the fundamental mode) as well as its higher harmonics is observed. We compared the normalized fluctuation amplitudes of the fundamental mode between potential and density, and obtained $\tilde{\Phi}_{\mathrm{f}} / \bar{T}_{\mathrm{e}}$ of $\sim 0.16$ and $\tilde{I}_{\mathrm{i}, \mathrm{sat}} / \bar{I}_{\mathrm{i} \text {,sat }}$ of $\sim 0.18$, which does not contradict the Boltzmann relationship. The fundamental mode has poloidal/axial mode numbers of $(m, n)=(3-5,2-3)[6]$, where $n=1$ means that the axial wave length is the same as the vessel length. In addition, the fundamental mode has a finite radial wave number in Fig. 1 (b). These properties of the fundamental mode do not contradict the estimation that the fundamental mode is the drift-wave [3].

Based on the Hasegawa-Mima equation (Eq. (8) in Ref. [6]), an energy transfer relationship in spectral form 


$$
\begin{aligned}
\phi=\sum_{\omega} \phi_{\omega} \text { is } & \\
\frac{\partial\left|\phi_{\omega}\right|^{2}}{\partial t} & =\frac{1}{2} \sum_{\omega=\omega_{1}+\omega_{2}} \Lambda_{\omega_{1}, \omega_{2}} \phi_{\omega_{1}} \phi_{\omega_{2}} \phi_{\omega}^{*}+\text { c.c. }, \\
\Lambda_{\omega_{1}, \omega_{2}} & =\frac{1}{1+\left(k_{\perp} \rho_{\mathrm{s}}\right)^{2}}\left(\boldsymbol{k}_{\omega_{1}} \times \boldsymbol{k}_{\omega_{2}}\right) \cdot \boldsymbol{e}_{z}\left[k_{\omega_{2}}^{2}-k_{\omega_{1}}^{2}\right],
\end{aligned}
$$

where $\phi$ is the normalized electrostatic potential, $\omega /(2 \pi)$ is the frequency, and $\boldsymbol{k}$ is the wave vector. The nonlinear three-wave coupling conditions, $\omega=\omega_{1}+\omega_{2}$ and $\boldsymbol{k}=\boldsymbol{k}_{1}+$ $\boldsymbol{k}_{2}$, where $\omega$ is the angular frequency of $\phi_{\boldsymbol{k}}$, are satisfied under the first order approximation. We estimate the first term of the right hand side of Eq. (1), the nonlinear energy transfer function, in frequency space. In addition, to calculate negative coupling conditions, $\omega=\omega_{1}-\omega_{2}$, we assume that a wave with a frequency of $-\omega$ has the wave vector $-\boldsymbol{k}_{\omega}$.

Figure 2 demonstrates an indicator of the energy transfer function, $F\left(\omega_{1}, \omega_{2}\right)=\left(\boldsymbol{k}_{\omega_{1}} \times \boldsymbol{k}_{\omega_{2}}\right) \cdot \boldsymbol{e}_{z}\left[k_{\omega_{2}}^{2}-\right.$ $\left.k_{\omega_{1}}^{2}\right] \mathfrak{R}\left\langle\phi_{\omega_{1}} \phi_{\omega_{2}} \phi_{\omega=\omega_{1}+\omega_{2}}^{*}\right\rangle$. Positive means that a mode with $\omega$ gains energy from coupling of modes with $\omega_{1}$ and $\omega_{2}$, and negative means the opposite. A two-dimensional contour of the transfer function is shown in Fig. 2 (a), and detailed slices in different $\omega_{2}$ axes are plotted in (b)-(d). The legend range from -0.05 to 0.05 in (a) is chosen to provide a clear view of transfer directions. We carefully calculated the transfer function in the frequency range where the aliasing effect of the wave number measurement can be eliminated.

In the positive frequency region of Fig. 2 (a) $(\omega \geq$ $\left.\omega_{1}, \omega_{2} \geq 0\right)$, a slice (b) is chosen along the $\omega_{2} /(2 \pi)=$ $7.14 \mathrm{kHz}\left(=\omega_{1 \mathrm{st}} /(2 \pi)\right.$, frequency of the fundamental mode), and we can see three positive spectral peaks at $\omega_{1}=2 \omega_{1 \mathrm{st}}, 3 \omega_{1 \mathrm{st}}$ and $4 \omega_{1 \mathrm{st}}$. The spectral peak at $\omega_{1}=2 \omega_{1 \text { st }}$ means that the mode with $3 \omega_{1 \text { st }}$ gains en-

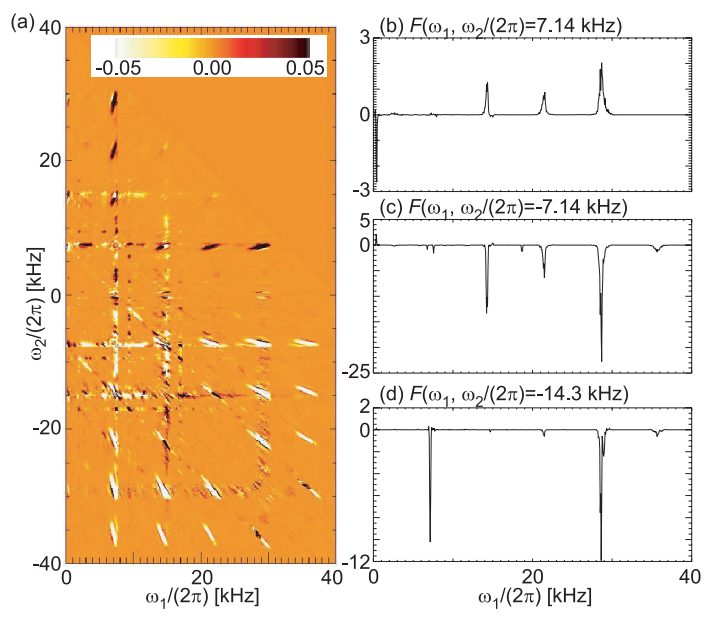

Fig. 2 Plots of an indicator of energy transfer function, $F\left(\omega_{1}, \omega_{2}\right)=\left(\boldsymbol{k}_{\omega_{1}} \times \boldsymbol{k}_{\omega_{2}}\right) \cdot \boldsymbol{e}_{z}\left[k_{\omega_{2}}^{2}-k_{\omega_{1}}^{2}\right] \mathfrak{R}\left\langle\phi_{\omega_{1}} \phi_{\omega_{2}} \phi_{\omega}^{*}\right\rangle$ [arb. unit]. $\omega=\omega_{1}+\omega_{2}$ holds. A two-dimensional plane (a), slices of (a) along lines at $\omega_{2} /(2 \pi)=7.14 \mathrm{kHz}$ (b), at $\omega_{2} /(2 \pi)=-7.14 \mathrm{kHz}(\mathrm{c})$ and at $\omega_{2} /(2 \pi)=-14.3 \mathrm{kHz}$ (d). ergy from mode coupling with $\omega_{1 \mathrm{st}}$ and $2 \omega_{1 \mathrm{st}}$. (This paper expresses the energy transfer relationship as $\phi\left(\omega_{1 \mathrm{st}}\right)+$ $\phi\left(2 \omega_{1 \mathrm{st}}\right) \Rightarrow \phi\left(3 \omega_{1 \mathrm{st}}\right)$. ) Other modes with $4 \omega_{1 \mathrm{st}}$ and $5 \omega_{1 \mathrm{st}}$ also gain energy from nonlinear couplings. On the contrary, the negative frequency region demonstrates many coherent negative peaks at the frequency of the higher harmonics of the drift-wave. Slices (c) or (d) are chosen along $\omega_{2}=-\omega_{1 \text { st }}$ or $\omega_{2}=-2 \omega_{1 \mathrm{st}}$. A spectral peak at $\omega_{1}=2 \omega_{1 \mathrm{st}}$ in (c) means that the mode with $\omega_{1 \mathrm{st}}$ releases energy to coupling of the modes with $-\omega_{1 \mathrm{st}}$ and $2 \omega_{1 \mathrm{st}}, \phi\left(\omega_{1 \mathrm{st}}\right) \Rightarrow \phi\left(2 \omega_{1 \mathrm{st}}\right)+\phi\left(-\omega_{1 \mathrm{st}}\right)$. We abbreviate the release process as Quasi-Parametric Decay Instability (QPDI). Other QPDIs show $\phi\left(2 \omega_{1 \mathrm{st}}\right) \Rightarrow \phi\left(3 \omega_{1 \mathrm{st}}\right)+\phi\left(-\omega_{1 \mathrm{st}}\right)$ and $\phi\left(3 \omega_{1 \mathrm{st}}\right) \Rightarrow \phi\left(4 \omega_{1 \mathrm{st}}\right)+\phi\left(-\omega_{1 \mathrm{st}}\right)$. The slice (d) also demonstrates a negative peak at $\omega_{1}=4 \omega_{1 \text { st }}$, indicating another QPDIs $\phi\left(2 \omega_{1 \mathrm{st}}\right) \Rightarrow \phi\left(4 \omega_{1 \mathrm{st}}\right)+\phi\left(-2 \omega_{1 \mathrm{st}}\right)$. Comparing transfer directions between positive and negative frequency region, both directions show forward cascades. For example, both processes of $\phi\left(2 \omega_{1 \mathrm{st}}\right) \Rightarrow \phi\left(3 \omega_{1 \mathrm{st}}\right)+\phi\left(-\omega_{1 \mathrm{st}}\right)$ and $\phi\left(\omega_{1 \mathrm{st}}\right)+\phi\left(2 \omega_{1 \mathrm{st}}\right) \Rightarrow \phi\left(3 \omega_{1 \mathrm{st}}\right)$ means that the second harmonics drive the third harmonics. For other coupling frequencies, the same relationship holds.

We discuss excitation of the second harmonics. In the Hasegawa-Mima equation, direct excitation of the second harmonic from coupling of the fundamental mode is prohibited. However, decay instability of a single $\omega$ mode may nonlinearly drive its second harmonics. Identification of the decay instability should be performed in measurement of the wave number spectrum, and we cannot conclude the QPDI in this manuscript. Other channels of second harmonics excitation are possible. Broadening the bandwidth of the fundamental mode produces two modes $\omega_{1} \pm \mathrm{d} \omega$, and couplings of the two modes may drive the second harmonics. However, this process is not clearly observed in this experiment. In addition, there could be a number of energy flow channels to the second harmonics by addressing various fluctuation fields such as density fluctuation [7]. Further research is necessary to determine the total energy channel to the second harmonics.

In summary, nonlinear energy transfer occurs from the fundamental drift-wave to its higher harmonics, and this confirms a forward cascading in the drift-wave spectrum. This energy transfer estimation is limited within the potential field, therefore, future investigations should consider energy transfer that includes the effects of various fluctuation fields as well as difference in location.

We are grateful to Prof. G.R. Tynan, Prof. P.H. Diamond, Prof. T. Ohkawa and Prof. F. Wagner for useful discussions. This work was partially supported by Grantin-Aids for SPR (16002005) [Itoh project] and for YS (B) (18760637) of MEXT, Japan, and by collaborations with NIFS (NIFS07KOAP017), RIAM, Kyushu Univ., and Univ. Tokyo. We also appreciate the support of Mr. T. Nishijima, Mr. M. Kawagughi and Mr. K. Kamataki. 
[1] W. Horton, Rev. Mod. Phys. 71, 735 (1999).

[2] Y. Saitou et al., Phys. Plasmas 14, 072301 (2007).

[3] Y. Nagashima et al., Proceedings on 35th European Physical Society on Plasma Physics (2008) P5.158.

[4] Y. Nagashima et al., J. Phys. Soc. Jpn. in press.
[5] Y. Nagashima et al., submitted to Phys. Rev. Lett.

[6] A. Hasegawa and K. Mima, Phys. Rev. Lett. 39, 205 (1977).

[7] M. Xu et al., Bulletin of APS meeting, Vol.52, 49th Division of Plasma Physics (2007) GP8.00017. 